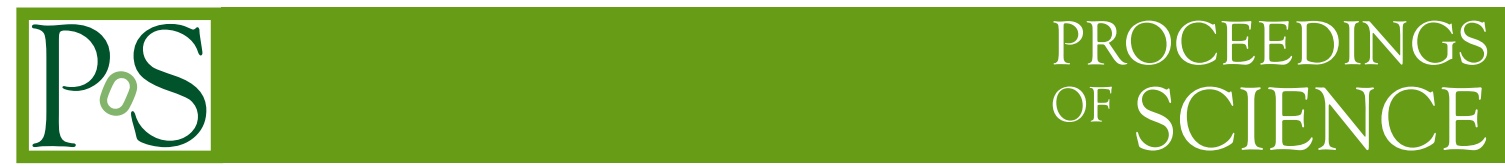

\title{
Origin of Cosmic Rays
}

\author{
Vladimir Ptuskin*独 \\ IPST, University of Maryland \\ E-mail: vptuskinehotmail.com
}

Brief overview of the current status and prospects of cosmic ray studies is presented

Frontiers of Fundamental Physics 14 - FFP14,

15-18 July 2014

Aix Marseille University (AMU) Saint-Charles Campus, Marseille, France

\footnotetext{
* Speaker.

${ }^{\dagger}$ The author is grateful to the organizers of the FFP14 Symposium for sponsorship and hospitality. The work was also supported by NASA grant NNX13AC46G and RFBR grant 13-02-00056
} 


\section{Introduction}

Our Galaxy and extragalactic space are filled with cosmic rays, a relativistic gas of high-energy protons, electrons, and heavy nuclei. The directly measured cosmic ray energy spectrum extends from about $1 \mathrm{MeV}$ to energies above $10^{20} \mathrm{eV}$. The radio-astronomical, X-ray, gamma-ray and the first very high energy neutrino observations shed light on the origin of cosmic rays. Below we touch up some problems of cosmic ray origin - the model of cosmic ray origin in supernova remnants (including pulsars), the interpretation of Voyager data on low energy particles, the structure of knee in cosmic ray spectrum at $3 \times 10^{15} \mathrm{eV}$, and the energy limit of Galactic sources. The origin of cosmic rays with energies above $10^{17}$ to $10^{18} \mathrm{eV}$ may be associated with the Active Galactic Nuclei, the progenitors of Gamma-Ray Bursts, the fast spinning newborn pulsars, the large-scale structure formation shocks and some other objects.

\section{2. "Golden age" of new cosmic ray measurements}

The last decade can be rightfully considered as a "golden age" of new cosmic-ray measurements. The list of remarkable experiments includes among other the Voyagers 1, 2 traveling throughout the outer heliosphere and the interstellar medium; ACE, PAMELA and AMS spacecraft missions; long-duration balloon experiments ATIC, BESS, CREAM and TRACER; IceCube Neutrino Observatory at the South Pole; the ground based Extensive Air Shower (EAS) detectors KASCADE-Grande, MILAGRO, ARGO-YBJ and Tunka; Auger and Telescope Array for studies of EAS produced by the highest-energy cosmic rays; Fermi gamma-ray space observatory and the ground based HESS, MAGIC and VERITAS telescopes for gamma-ray observations of cosmic ray sources. The references to the experimental results of cosmic ray research can be found in [四]. The detection of astrophysical neutrinos with $\mathrm{PeV}$ and sub-PeV energies in the IceCube experiment [0] has opened the new way to a study of the sources of very high energy cosmic rays. The detected neutrino events are scattered over the sky without evident correlation with any Galactic or extragalactic classes of astronomical objects.

Cosmic rays of the highest energies continue to hold the great interest of physicists. The HiRes, Auger and Telescope Array installations experimentally established the suppression of cosmic ray flux starting at energy about $5 \times 10^{19} \mathrm{eV}$ as expected due to the Greisen-Zatsepin-Kuzmin effect, the energy loss of the highest energy cosmic rays interacting with the extragalactic microwave and optic background radiation, see review [3]. The development of detailed models of acceleration and propagation of the highest energy cosmic rays is hampered by the uncertainty of the mass composition. The interpretation of HiRes and Telescope Array data favours the proton composition at energies $10^{18}$ to $5 \times 10^{19} \mathrm{eV}$, whereas the Auger data indicate that cosmic ray composition is becoming heavier with energy changing from predominantly proton at $10^{18} \mathrm{eV}$ to more heavy and probably reaching the pure iron composition at about $5 \times 10^{19} \mathrm{eV}$.

Launched in 1977 and expected to operate till 2020-2025, Voyager 1 is 130 a. u. from the Sun and Voyager 2 is at 107 a. u.. Voyager 1 is in "interstellar space" and Voyager 2 is currently in the "heliosheath" - the outermost layer of the heliosphere where the solar wind is slowed by the pressure of interstellar gas. The Voyager 1 spacecraft is measuring cosmic ray intensity not affected by the solar wind modulation. This experiment extends our knowledge of the local interstellar 
cosmic ray spectrum and composition down to $\mathrm{MeV}$ energies [ [ 9 ]. The spectra of protons, nuclei and electrons look as a natural continuation of spectra at energies above a few hundred $\mathrm{MeV}$ and no any additional low energy component was revealed. The intensity of the so called Anomalous Cosmic Rays (ACR) is diminished to zero and it confirms that these particles are accelerated somewhere in the inner part of the heliosheath (probably, at the hot spots of the solar wind termination shock).

The remarkable result of PAMELA experiment was the detection of unexpectedly large flux of positrons with energies above $10 \mathrm{GeV}$ [5]. This result was confirmed by the AMS-02 experiment [6] The measured flux of positrons is larger than the calculated flux of secondary positrons produced in cosmic ray interactions with interstellar gas atoms. The high content of positrons can be explained by the contribution of high-energy electron-positron pairs generated by pulsars or by the acceleration of positrons in the reverse shocks moving through the radioactive ejecta in supernova remnants. The conclusive elucidation of the nature of positron flux in cosmic rays is very important since an alternative explanation suggests that these positrons are the products of dark matter decay.

Recent high-accuracy ATIC-2, CREAM and PAMELA measurements revealed deviations of the cosmic ray spectrum from a plain power law at energies from 10 to $10^{5} \mathrm{GeV} / \mathrm{n}$ [Q], \&]. These deviations include the spectral hardening in proton, helium and nuclear spectra at magnetic rigidity $\sim 240 \mathrm{GV}$ and an increase of the He/p ratio with energy. One of the explanations of these features implies cosmic ray acceleration by reverse shocks in supernova remnant [Q] . New experimental results require the improvement of existing models of cosmic ray acceleration and propagation in the Galaxy although the basic model with the cosmic ray acceleration in supernova remnants (including pulsars) followed by the propagation in galactic magnetic fields with a slow leakage from the Galaxy remains immutable.

The fine structure of the all particle cosmic ray spectrum at $10^{5} \mathrm{GeV}$ to $10^{9} \mathrm{GeV}$ and the data on elemental composition were clarified in the KASCADE-Grande and Tunka experiments [एU, प]. This structure corresponds to the superposition of fluxes of different nuclei with similar spectra on magnetic rigidity and the breaks (knees) at $3 \times 10^{6} \mathrm{Z} \mathrm{GV} \mathrm{(} \mathrm{Z}$ is the particle charge). It results in the all-particle spectrum with a proton-helium knee at $3 \times 10^{6} \mathrm{GeV}$ and the steepening of a power law energy spectrum after this energy. The second knee at energy just below $10^{17}$ $\mathrm{eV}$ where composition is dominated by heavy (iron) nuclei was found. The hard light component emerge at about $10^{17} \mathrm{eV}$. This component may be of Galactic origin or reflect the transition to the extragalactic cosmic rays.

Efforts of theorists in the last few decades were mainly focused on the problem of particle acceleration by shocks propagating in the turbulent interstellar plasma [12, [13]. The modeling of nonlinear shock acceleration in supernova remnants demonstrated that the calculated energy spectrum of cosmic rays after propagation through the Galaxy is in good agreement with the measured spectrum up to approximately $5 \times 10^{18} \mathrm{eV}$ [ㄴ]].

\section{Prospects}

After many years of cosmic ray research we have a well-developed basic model of cosmic ray origin but a number of open questions require further investigation. Among them are the search for indirect evidence for dark matter through features in cosmic ray and gamma ray spectra, the determination of the energy limit of galactic sources and the transition from galactic to extragalactic 
component in the observed cosmic ray spectrum, the identification of sources and acceleration mechanisms of the highest energy cosmic rays.

Further progress in understanding of these and other problems of cosmic ray astrophysics will be achieved with the help of new theoretical efforts and the ambitious experiments planned for the next few years, such as ISS-CREAM where the CREAM instrument will be delivered to the International Space Station by the Space-X vehicle and will directly study cosmic ray composition up to the knee, CALET (CALorimeteric Electron Telescope) is a project for the International Space Station to study electrons, protons and higher-energy nuclei, JEM-EUSO (Extreme Universe Observatory) for measuring cosmic rays with energies $\geq 3 \times 10^{19} \mathrm{eV}$ from space, LHAASO (the multi-parameter Large High Altitude Air Shower Observatory) in Tibet for investigations of veryhigh energy gamma rays and cosmic rays, CTA (Cherenkov Telescope Array) of 100 telescopes to study $100 \mathrm{GeV}$ to $100 \mathrm{TeV}$ gamma rays, the HiSCORE (Hundred Square-km Cosmic Origin Explorer) instrument will be constructed at the Tunka site in Siberia and it will use open Cherenkov counters to measure cosmic rays around the knee, ANITA (Antarctic Impulsive TRansient Antenna) experiment is designed to study ultra-high-energy cosmic neutrinos in the Antarctic ice through the Askaryan effect. The discussion of some current and planned cosmic ray experiments can be found in [15]].

\section{References}

[1] K.A. Olive et al. (Particle Data Group), Review of particle physics, Chinise Phys. C 38 (2014) 090001.

[2] IceCube Collaboration, Evidence for high-energy extraterrestrial neutrinos at the IceCube detector, Science 342 (2013) 1242856.

[3] K. Kotera, A. V. Olinto, The astrophysics of ultrahigh-energy cosmic rays, Annu. Rev. Astron. Astrophys. 49 (2011) 119.

[4] E. Stone et al., Voyager 1 observes low-energy Galactic cosmic rays in a region depleted of heliospheric ions, Science 341 (2013) 150.

[5] O. Adriani et al., An anomalous positron abundance in cosmic rays with energies $1.5-100 \mathrm{GeV}$, Nature 458 (2009) 607.

[6] M. Aguilar et al., Electron and positron fluxes in primary cosmic rays measured with Alpha Magnetic Spectrometer on the International Space Station, Phys. Rev. Lett. 113 (2014) 121102.

[7] O. Adriani et al., PAMELA measurements of cosmic-ray proton and helium spectra, Science 332 (2011) 69.

[8] H.S. Ahn et al., Discrepant hardening observed in cosmic-ray elemental spectra, ApJ Lett. 714 (2010) L89.

[9] V.S. Ptuskin, V.N. Zirakashvili, E.S. Seo, Spectra of cosmic-ray protons and helium produced in supernova remnants, ApJ 763 (2013) 47.

[10] A. Haungs et al., High-energy cosmic rays measured with KASACADE-Grande, in proceedings of 33rd Intern. Cosmic Ray Conf., Rio de Janeiro (2013) in press [arXiv:1308.1485v1].

[11] V.V. Prosin et al., Tunka-133: results of 3 year operation, Nucl. Instrum. Methods in Physics Research A 756 (2014) 94. 
[12] A.R. Bell, Cosmic ray acceleration, Astropart. Physics 43 (2013) 56.

[13] D. Caprioli, Hybrid simulations of particle acceleration at shocks, Nucl. Phys. B (Proc. Suppl. 256 (2014) 48

[14] V.S. Ptuskin, V.N. Zirakashvili, E.S. Seo, Spectrum of Galactic cosmic rays accelerated in supernova remnants, ApJ 718 (2010) 31.

[15] Z. Cao, J. Lui, Cosmic Ray experiments in the Era of LHC, in proceedings of 25th Texas Symposium on Relativistic Astrophysics, AIP Conf. Proc. 1381, American Institute of Physics, Melville, NY (2011) 159. 